\title{
E-cadherin mediated cellular effects and molecular partners. Gastric cancer as an invasive model
}

\author{
Joana Figueiredo, Joana Simões-Correia, Joana Fernandes, Ana R Mateus, Gianpaolo Suriano, Joana Paredes, \\ Carla Oliveira, Raquel Seruca* \\ From 16th International Charles Heidelberger Symposium on Cancer Research \\ Coimbra, Portugal. 26-28 September 2010
}

Germline mutations of E-cadherin gene (CDH1) occur in 30\% of families with Hereditary Diffuse Gastric Cancer (HDGC); of these, 23\% are missense mutations. The CDH1 missense mutations described to date span the entire gene and most of them are functionally relevant and constitute a invaluable model do study E-cadherin dependent signaling pathways and find new molecular targets for therapy. Recently, we explored the hypothesis that mutations affecting different E-cadherin protein domains have distinct effects on cell motility. To verify this hypothesis we characterized the effect of eleven HDGC CDH1 germline missense mutations (T118R, L214P, G239R, A298T, T340A, P373L, R749W, E757K, E781D, P799R and V832M) on cell motility. Further, we studied their effect on the activation of signaling pathways known to be relevant for cell motility, such as the EGFR signaling.

We found that all cell lines expressing extracellular mutant forms of E-cadherin (T118R, L214P, G239R, A298T and P373L) displayed higher EGFR activation than cells expressing wild-type E-cadherin. This phenotype is similar to what we observed for cells negative for E-cadherin, which also displayed increased EGFR phosphorylation in comparison to the wild-type expressing cells. The same pattern was observed for the cells expressing the intracellular mutations within the juxtamembrane region, R749W and E757K, which is in accordance with the motility analysis results. Our results allowed the identification of the E-cadherin domains pivotal for cell motility, further demonstrated a genotype-phenotype correlation, and defined a subset of HDGC cases which may benefit from EGFR inhibitors.

\footnotetext{
* Correspondence: rseruca@ipatimup.pt

IPATIMUP- Institute of Molecular Pathology and Immunology of the

University of Porto, Porto, Portugal

Full list of author information is available at the end of the article
}

The role of E-cadherin in tumor development is well established, with many human carcinomas exhibiting E-cadherin loss at the invasive front. In many invasive carcinomas, the mechanisms leading to the loss of E-cadherin remains elusive. We recently hypothesize that trafficking deregulation has a role in the regulation of E-cadherin missense mutations. We first analyzed the intracellular trafficking of two HDGC-associated missense mutations, R749W and E757K, localized in the justamembranar domain of E-cadherin, know to have higher impact in E-cadherin trafficking. We found that these mutations frequently accumulate in the endoplasmic reticulum. Moreover, treatment of the mutant cells with specific chemical chaperones, restored mutant E-cadherin to the cell membrane and rescued its function. The protein-stabilizing power of chemical chaperones has been show to be effective on rescuing different disease-associated mutant proteins, but the mechanism is not understood. In order to understand and further explore the therapeutic power of such organic molecules in the context of HDGC, be believe its crucial to dissect their mode of action in the regulation of E-cadherin mutants. We are now investigating the mechanism by which the chemical chaperone DMSO rescues mutant E-cadherin expression by analyzing its effect on different known E-cadherin trafficking regulators.

Published: 24 September 2010

\section{doi:}

Cite this article as: Figueiredo et al:: E-cadherin mediated cellular effects and molecular partners. Gastric cancer as an invasive model. BMC Proceedings 2010 4(Suppl 2):O22. 